\title{
The Pharmacological Effects of Plant- Derived versus Synthetic Cannabidiol in Human Cell Lines
}

\author{
Ryan F. Maguire ${ }^{a}$ Daniel J. Wilkinson ${ }^{a}$ Timothy J. England ${ }^{a}$ b \\ Saoirse E. O'Sullivan ${ }^{a, c}$ \\ aDivision of Graduate Entry Medicine and Medical Sciences, School of Medicine, University of Nottingham, Royal \\ Derby Hospital, Nottingham, UK; ${ }^{\text {b}}$ Department of Stroke, University Hospitals of Derby and Burton, Derby, UK; \\ 'Artelo Biosciences, Inc., La Jolla, CA, USA
}

\section{Keywords}

Cannabidiol · Synthetic cannabidiol · Plant · Pharmacology · Cannabinoid receptor $1 \cdot 5 \mathrm{HT}_{1 \mathrm{~A}}$

\begin{abstract}
Introduction: Cannabidiol (CBD) can be isolated from Cannabis sativa $\mathrm{L}$. or synthetically produced. The aim of this study was to compare the in vitro effects of purified natural and synthetic CBD to establish any pharmacological differences or superiority between sources. Methods: Six purified samples of CBD were obtained, 4 of these were natural and 2 synthetic. The anticancer effects of $C B D$ were assessed in a human ovarian cancer cell line (SKOV-3 cells). The neuroprotective effects of $C B D$ were assessed in human pericytes in a model of stroke (oxygen glucose deprivation [OGD]). The ability of $C B D$ to restore inflammation-induced intestinal permeability was assessed in differentiated human Caco-2 cells (a model of enterocytes). Results: (1) In proliferating and confluent SKOV-3 cells, all CBD samples similarly reduced resazurin metabolism as a marker of cell viability in a concentration-dependent manner $(p<0.001)$. (2) In pericytes exposed to OGD, all CBD samples similarly reduced cellular damage (measured by lactate dehydrogenase) at $24 \mathrm{~h}$
\end{abstract}

karger@karger.com www.karger.com $/ \mathrm{mca}$

Karger"

GOPEN ACCESS
(C) 2021 The Author(s)

Published by S. Karger AG, Basel

This is an Open Access article licensed under the Creative Commons Attribution-NonCommercial-4.0 International License (CC BY-NC) (http://www.karger.com/Services/OpenAccessLicense), applicable to the online version of the article only. Usage and distribution for commercial purposes requires written permission. by $31-48 \%$ and reduced inflammation (measured by IL- 6 secretion) by $30-53 \%$. Attenuation of IL- 6 was inhibited by $5 \mathrm{HT}_{1 \mathrm{~A}}$ receptor antagonism for all $\mathrm{CBD}$ sources. (3) In differentiated Caco-2 cells exposed to inflammation (TNFa and IFNy, $10 \mathrm{ng} / \mathrm{mL}$ for $24 \mathrm{~h}$ ), each CBD sample increased the speed of recovery of epithelial permeability compared to control $(p<0.05-0.001)$, which was inhibited by a $\mathrm{CB}_{1}$ receptor antagonist. Conclusion: Our results suggest that there is no pharmacological difference in vitro in the antiproliferative, anti-inflammatory, or permeability effects of purified natural versus synthetic CBD. The purity and reliability of $\mathrm{CBD}$ samples, as well as the ultimate pharmaceutical preparation, should all be considered above the starting source of $C B D$ in the development of new CBD medicines.

$$
\begin{aligned}
& \text { C } 2021 \text { The Author(s). } \\
& \text { Published by S. Karger AG, Basel }
\end{aligned}
$$

\section{Introduction}

Preclinical and early clinical phase studies demonstrate the potential of cannabidiol (CBD) in cancer, stroke, anxiety, and pain [1-8], and a purified form of CBD $\left(98 \%\right.$, Epidiolex $\left.{ }^{\circledR}\right)$ is already licensed to treat sei- 
zures associated with Dravet syndrome, Lennox-Gastaut syndrome, and tuberous sclerosis [9]. Around 65 molecular targets for CBD have been identified $[10,11]$ with different targets responsible for different therapeutic effects of CBD. For example, serotonin $1 \mathrm{~A}$ receptor $\left(5 \mathrm{HT}_{1 \mathrm{~A}}\right)$ activation is associated with reductions in anxiety, nausea, and neuroprotective effects [12-14]. Transient receptor potential cation channel subfamily V member 1 (TRPV1) interactions have been implicated in the antinociceptive effects of CBD [15]. Peroxisome proliferator-activated receptor gamma (PPAR $\gamma$ ), cyclooxygenase-2 (COX-2), and GPR55 have been linked to the anticancer effects of CBD [16]. Cannabinoid receptor $1\left(\mathrm{CB}_{1}\right)$ interactions have been linked with CBD's ability to reduce intestinal permeability $[17,18]$. The polypharmacology of CBD may underpin its ability to affect many pathologies.

Over-the-counter CBD products are mainly produced from Cannabis sativa L. with a THC content below $0.2 \%$ (hemp). Epidiolex ${ }^{\circledR}$ is produced using C. sativa L. with a higher THC content. CBD can also be produced synthetically through a series of chemical reactions, yielding a highly pure form of CBD [19]. Natural CBD products are either refined to get purified CBD (97-99\%), or crude extraction methods are used to yield CBD alongside other phytocannabinoids, terpenes, and flavonoids, which is known as a "CBD-rich extract."

One complication that can arise in reviewing the effectiveness of CBD medicines is this range of products that include purified isolate forms or extracts containing other chemicals. Some suggest that CBD-rich extracts are therapeutically superior because of the "entourage" hypothesis. Scientific evidence, however, is equivocal. Gallily and colleagues [20] showed CBD-rich extracts were more effective than purified CBD in attenuating inflammation and hyperalgesia in rats. Similarly, Pagano et al. [21] showed a CBD-rich extract was more beneficial in attenuating intestinal inflammation and hypermobility in mice. In contrast, Scott et al. [22] found purified CBD was more efficacious than a CBD-rich extract in glioblastoma cell lines. Ligresti and colleagues [23] also found purified $\mathrm{CBD}$ was more potent in reducing proliferation in some cancer cell lines, while in others, the CBD-rich extract was more potent. Similarly, Raup-Konsavage and colleagues [24] found that pure CBD was more efficacious than 3 different CBD extracts in reducing cancer cell viability. Thus, it is not yet clear whether purified or CBD extracts are superior, or whether this is different depending on the pathological situation in which CBD is being tested. There are also no controlled clinical trial data to support either argument.

Are All CBDs the Same?
Because of the perception that $\mathrm{CBD}$ extracts are superior to purified $\mathrm{CBD}$, synthetic CBD products are sometimes perceived as inferior. A recent study in Germany asked 153 epileptic patients whether they worry about the origin of CBD. Seventy-three percent favored natural CBD [25]. The main reasons were a preference for a botanical origin, a perceived lack of toxicity relative to synthetic forms, and an absence of chemical reactions in the manufacturing process. Conversely, there is also a perception that contaminations of natural CBD products (such as pesticides, heavy metals, microbial pathogens, and carcinogenic compounds) during cultivation, manufacturing, and packaging may limit the pharmaceutical use of natural CBD [26]. There are no head-to-head clinical studies comparing plant versus synthetic CBD. However, studies assessing the efficacy, safety, and pharmacokinetic parameters of synthetic CBD in drugresistant epilepsy $[27,28]$ found very similar characteristics relative to natural CBD [29-32].

In light of the lack of data directly comparing purified CBD of various origins, this study aimed to establish whether the CBD source affects its pharmacological effects in human cells. Our hypothesis was that there would be no difference in the effects produced between CBD samples of similar purity.

\section{Methods}

\section{Materials}

CBD derived from 2 different sources ( 4 natural and 2 synthetic) were obtained from 6 companies (see Table 1). Only Logical confirmed to use a synthesis process selective for the naturally occurring (-) enantiomer of CBD. For natural CBD, manufacturers' analysis demonstrated Medropharm (Medropharm $\mathrm{GmbH}$ ) was 98.6\% CBD, 0.2\% CBD-C4 analog, 0.6\% cannabidivarin (CBD-C3, $\mathrm{CBDV}$ ), and $0.3 \%$ cannabigerol. Flora Fusion was $99.3 \% \mathrm{CBD}$ and 0.2\% CBDV. CBDepot was 98.7\% CBD, 0.3\% CBD-C4, and $0.6 \%$ CBDV. Ai Lab was 99.4\% CBD and had no data regarding any impurities. Of the synthetic CBD, Logical was $98.9 \%$ and THC Pharm was $99.8 \%$ pure. The presence of impurities was not assessed in either synthetic CBD sample. CBD was in ethanol at $10^{-1} \mathrm{~mol} / \mathrm{L}$. For each experimental repeat, a new stock solution was made.

Antagonists of receptors known to mediate CBD effects were used at relevant concentrations: $\mathrm{CB}_{1}$ receptor antagonist, $\mathrm{AM} 251$ $(100 \mathrm{nM}) ; \mathrm{CB}_{2}$ receptor antagonist, AM630 (100 nM); GPR18 antagonist, O-1918 (1 $\mu \mathrm{M})$; GPR55 antagonist, CID16200 (5 $\mu \mathrm{M})$; PPARa antagonist, GW6471 (100 nM); PPAR $\gamma$ antagonist, GW9662 (100 nM); TRPV1 receptor antagonist, SB-36679 (1 $\mu \mathrm{M})$; and $5 \mathrm{HT}_{1 \mathrm{~A}}$ antagonist, WAY-100635 (300 nM). All antagonists were from Tocris (Abingdon, UK). When used, antagonists were applied $20 \mathrm{~min}$ before CBD.

\section{Cell Culture}

Cells were maintained in a humidified cell culture incubator at $37^{\circ} \mathrm{C}$ with $5 \% \mathrm{CO}_{2}$ and cultured to $70-80 \%$ confluence in a $\mathrm{T} 75$ 


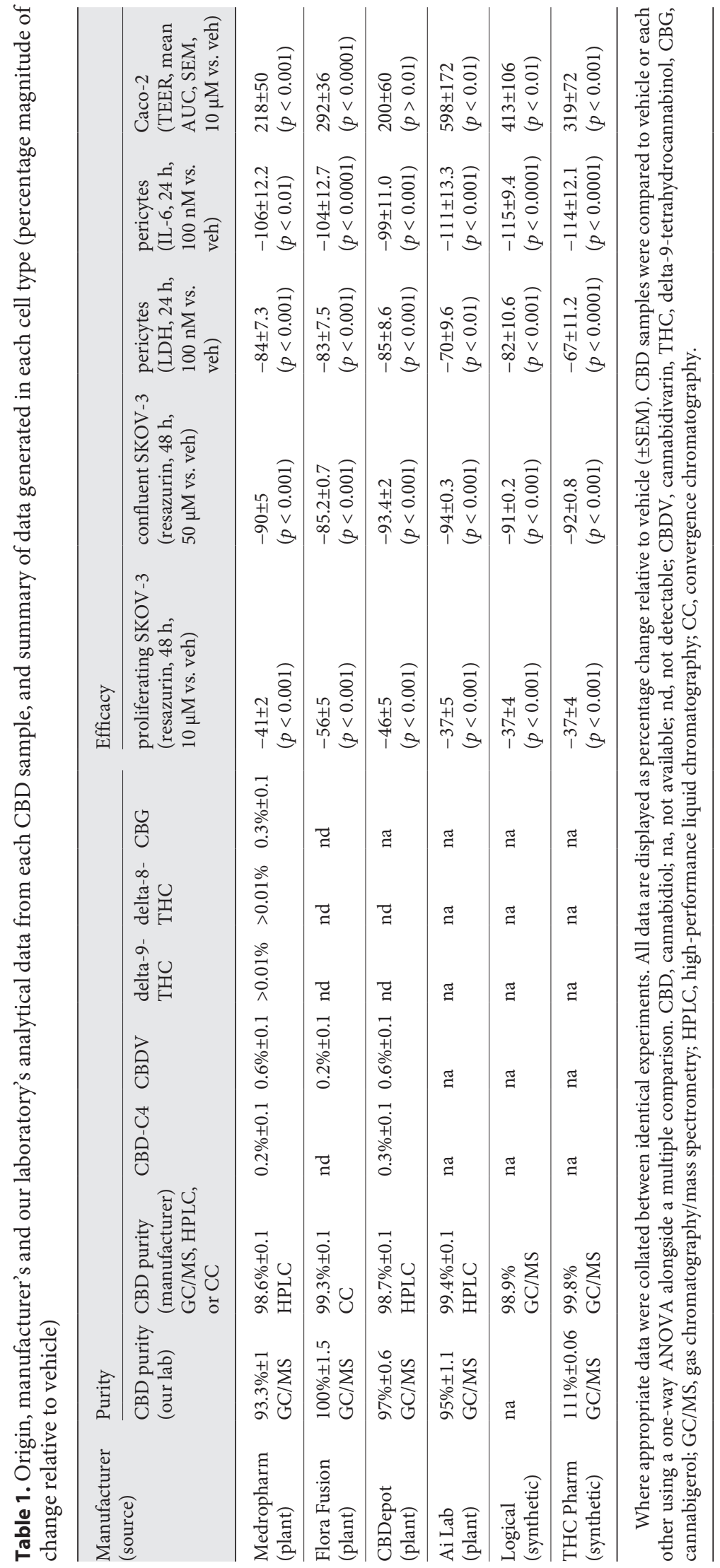


flask. SKOV-3 (The European Collection of Authenticated Cell Cultures [ECACC, Salisbury, UK], passages 24-31) cell lines were cultured to $70-80 \%$ confluence in Roswell Park Memorial Institute (RPMI) 1640 base medium containing L-glutamine and phenol red indicator (Gibco), supplemented with 10\% heat-inactivated FBS and $1 \%$ penicillin-streptomycin $(\mathrm{P} / \mathrm{S})$. Human brain vascular pericytes (ScienceCell, Carlsbad, CA, USA, passages 3-6) were cultured in specialized pericyte medium $(2 \% \mathrm{FBS}, 1 \%$ pericyte growth supplement, and 1\% P/S; ScienceCell, Carlsbad, CA, USA). Caco-2 cells (ECACC, Salisbury, UK) (passages 37-49) were cultured in Minimum Essential Medium Eagle (1\% P/S, 1\% nonessential amino acids, and 10\% FBS).

\section{Gas Chromatography/Mass Spectrometry}

Test stock solutions of each CBD compound were made up at $50 \mu \mathrm{g} / \mathrm{mL}$ by weight in methanol. Twenty microliters of $10 \mu \mathrm{g} / \mathrm{mL}$ D3-CBD (Sigma-Aldrich, Poole, UK) was added to $100 \mu \mathrm{L}$ of standard and test stock solution, mixed, and evaporated until dry under nitrogen at $30^{\circ} \mathrm{C}$. Samples and standards were derivatized to their CBD-TMS esters by adding $70 \mu \mathrm{L}$ of acetonitrile and $70 \mu \mathrm{L}$ of BSTFA (Apollo Scientific, Manchester, UK) and incubating for 90 min at $90^{\circ} \mathrm{C} .0 .5 \mu \mathrm{L}$ of each sample and standard was injected into a Trace 1310-TSQ 8000 GC-MS/MS (Thermo Scientific, Hemel Hempstead, UK) in SRM mode monitoring $\mathrm{m} / \mathrm{z}$ transitions 390319 for unlabeled CBD and 393-319 for D3-labeled CBD. Concentrations of test stock solutions were calculated from the standard curve of known CBD concentrations ranging from 20 to $80 \mu \mathrm{g} / \mathrm{mL}$ and \% recovery calculated. Linearity of standard curves was assessed for each assay and accepted if $R^{2}>0.97$. CV for repeat sample injections was $1 \%$.

\section{Anticancer Effects of CBD in SKOV-3 Cells}

To identify the concentration of CBD to use in studies with SKOV-3 cells, a concentration-response curve was constructed with CBD from THC Pharm (see online suppl. Fig. 1; for all online suppl. material, see www.karger.com/doi/10.1159/0005171203). SKOV-3 cells were seeded into 96-well plates at a density of 10,000 cells $/ \mathrm{cm}^{2}$ and left to adhere for $3 \mathrm{~h}$. Media were replaced with fresh media containing either vehicle (ethanol) or CBD (10-50 $\mu \mathrm{M})$. Plates were allocated into 2 time points: 24 and $48 \mathrm{~h}$. At each time point, resazurin (Sigma-Aldrich, Poole, UK) dissolved in PBS (Thermo-Fisher, UK) was added to the wells to produce an in-well concentration of $100 \mu \mathrm{M}$ and returned to the incubator for $2 \mathrm{~h}$. Plates were read using a Fluoroskan Ascent microplate fluorometer (Thermoelectron Corporation, Waltham, MA, USA) at $560 \mathrm{~nm}$ excitation and $590 \mathrm{~nm}$ emission. Ten and $50 \mu \mathrm{M}$ were identified as subeffective and effective concentrations for similar experiments comparing the different CBD samples.

In separate experiments, the cytotoxic effect of CBD was investigated using confluent SKOV-3 cells. Cells were seeded into 96well plates and given 2 days to reach confluence. The media were aspirated and replaced with fresh media either containing ethanol (vehicle) or CBD (10 or $50 \mu \mathrm{M})$ for 24 or $48 \mathrm{~h}$ and resazurin metabolism measured as before.

Oxygen Glucose Deprivation Experiments in Pericytes

Human pericytes were exposed to oxygen glucose deprivation/ reperfusion $(\mathrm{OGD} / \mathrm{R})$ as a model of stroke [33, 34]. Cells were seeded at 20,000 cells $/ \mathrm{cm}^{2}$ in a 96-well plate. Once confluent, cells were incubated for $24 \mathrm{~h}$ to establish baseline samples. The media were replaced with RPMI glucose-free media (Invitrogen, Carlsbad, CA, USA), containing either CBD (10 or $100 \mathrm{nM}$ ) or vehicle (ethanol) based on our unpublished work. Plates were sealed into GasPak EZ Anaerobe Pouches (Beckton Dickinson, Oxford, UK) and placed into the incubator, producing anoxic conditions within $20 \mathrm{~min}$ [33]. After $4 \mathrm{~h}$, media were collected and replaced with complete pericyte growth medium containing either CBD (10 or $100 \mathrm{nM}$ ) or ethanol (vehicle) and returned to normoxic conditions for $20 \mathrm{~h}$. Media concentrations of IL-6 (immediately after OGD and $24 \mathrm{~h}$; R\&D systems ELISA) and lactate dehydrogenase (LDH, $24 \mathrm{~h}$ only; Abcam) were measured as per manufacturer's instructions.

Inflammation and Permeability Experiments in Caco-2 Cells

Caco-2 cells (P32-45) were seeded onto the apical compartments of polycarbonate transwell inserts (12-mm diameter, 0.4$\mu \mathrm{m}$ pore diameter; Transwell; Corning Inc., Corning, NY, USA) at a density of 20,000 cells $/ \mathrm{cm}^{2}$ with complete medium. Cells were differentiated over 21 days into gut enterocyte-like cells. Transepithelial electrical resistance (TEER, ohms, $\Omega$ ) was used to quantify barrier integrity using STX2 chopstick electrodes attached to an Ohm meter (World Precision Instruments, Hitchin, UK) [35]. Wells with a resistance of over $999 \Omega$ were used for experiments. The basolateral compartments of each well were treated with 10 $\mathrm{ng} / \mathrm{mL}$ IFN $\gamma$, and $8 \mathrm{~h}$ later, with $10 \mathrm{ng} / \mathrm{mL}$ TNFa. Sixteen hours later, TEER was measured and the wells washed once with PBS. Fresh media containing CBD $(10 \mu \mathrm{M})$ were applied to the apical membrane and TEER measured up to $72 \mathrm{~h}$.

\section{Data Analysis}

Statistical analyses were performed using GraphPad Prism 8.2.0 and assessed for Gaussian distribution prior to analysis. Resazurin, LDH, and IL-6 datasets were analyzed using one-way ANOVA alongside a Dunnett's post hoc test comparing CBD samples against vehicle controls, as well as a multiple comparison comparing each $\mathrm{CBD}$ sample at the respective concentrations and time points. Caco-2 time course data were analyzed using area under the curve (AUC), quantified using the trapezoidal method, and compared to vehicle using an unpaired $t$ test. AUC data were compared by the CBD sample using a one-way ANOVA multiple comparison with a Dunnett's post hoc test.

\section{Results}

\section{Gas Chromatography/Mass Spectrometry}

In general, the purity of each CBD was lower for our laboratory's measurements than the manufacturers' (Table 1). This could be due to different measurement techniques and/or degradation over time. Regardless, the purity of the CBD samples was independently confirmed in our lab using GC/MS. Logical CBD suffered from a poor percentage recovery in both runs, and unfortunately, the particular batch of Logical CBD used in the cellular experiments had been exhausted following these runs, so we were unable to obtain purity data with this sample. 


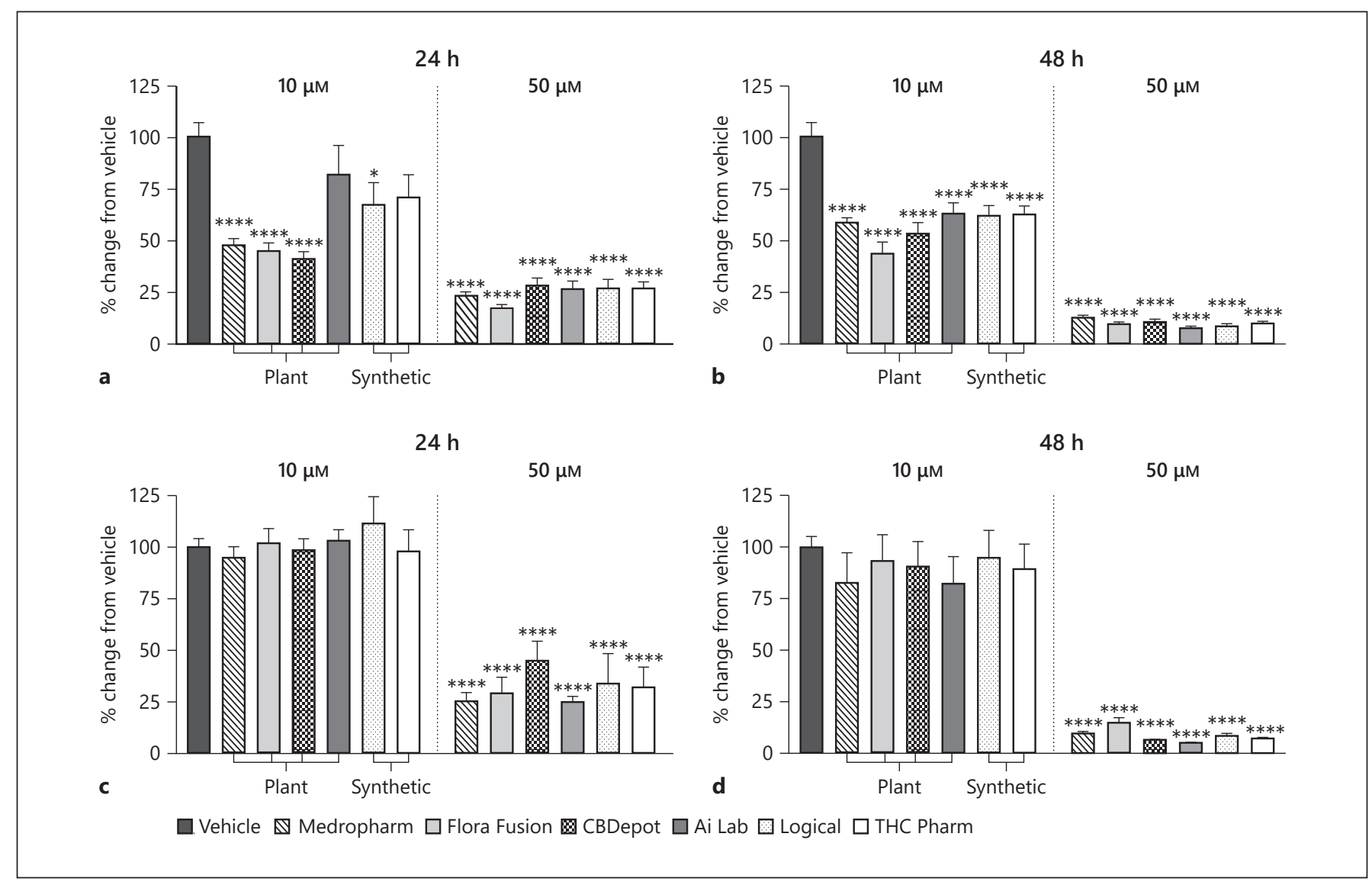

Fig. 1. Effects of various $\mathrm{CBD}$ samples on SKOV-3 cellular metabolism using resazurin assays. CBD $(10$ or $50 \mu \mathrm{M})$ was applied to proliferating SKOV -3 cells for 24 (a) and 48 h (b) $(n=15$ from 3 separate experiments). Effects of CBD (10 and $50 \mu \mathrm{M})$ on confluent SKOV -3 cells applied for 24 (c) and $48 \mathrm{~h}(\mathbf{d})(n=10$ from 2 sepa- rate experiments). Data are displayed as mean \pm SEM \% change from vehicle and were compared for statistical significance against vehicle using a one-way ANOVA. ${ }^{*} p \leq 0.05 ;{ }^{* * * *} p \leq 0.0001 . \mathrm{CBD}$, cannabidiol.

\section{Anticancer Effects of Natural and Synthetic CBD in SKOV-3 Cell Line}

In proliferating SKOV-3 cells, at $24 \mathrm{~h}$ (Fig. 1a), $10 \mu \mathrm{M}$ Medropharm $(-53 \% \pm 6)$, Flora Fusion $(-55 \% \pm 6)$, and CBDepot $(-59 \% \pm 6)$ reduced resazurin metabolism relative to vehicle control $(p<0.0001)$. Logical CBD also reduced resazurin metabolism, albeit to a lesser extent $(-33 \% \pm 9, p<0.05$; Fig. 1a). At 24 or 48 h, $50 \mu \mathrm{M}$ of all CBD sources produced similar reductions in resazurin metabolism compared to vehicle ( $p<0.0001$; Fig. 1a, b). No statistically significant differences existed between CBD samples.

In fully confluent SKOV-3 cells, after $24 \mathrm{~h}$, only $50 \mu \mathrm{M}$ $\mathrm{CBD}$ reduced resazurin metabolism relative to vehicle $(-55$ to $-74 \%, p<0.0001$; Fig. 1c). Similarly, after 48 h, only $50 \mu \mathrm{M} \mathrm{CBD}$ reduced resazurin metabolism relative to vehicle ( -85 to $-95 \%, p<0.0001$; Fig. 1 d). No significant differences were observed at either concentration or time point between CBD samples. None of the selected antagonists (to $5 \mathrm{HT}_{1 \mathrm{~A}}, \mathrm{CB}_{1}, \mathrm{CB}_{2}$, GPR18, GPR55, PPARa, PPAR $\gamma$, and TRPV1 receptors) inhibited the reduction in metabolism associated with CBD (Fig. 2).

\section{The Effects of CBD in Pericytes Exposed to OGD}

IL- 6 content immediately after OGD was $225 \% \pm 63$ $(p<0.05$; Fig. 3a) higher than in normoxic control cells and at $24 \mathrm{~h}$ was $184 \% \pm 26$ higher $(p<0.01$; Fig. 3b). At $24 \mathrm{~h}, \mathrm{LDH}$ activity in media was $118 \% \pm 18$ higher than normoxic control cells ( $p<0.01$; Fig. $3 c)$.

IL-6 secretion immediately after OGD was not significantly raised in the presence any of the CBDs at 10 or 100 nM (Fig. 3a). IL-6 secretion at $24 \mathrm{~h}$ following OGD was 


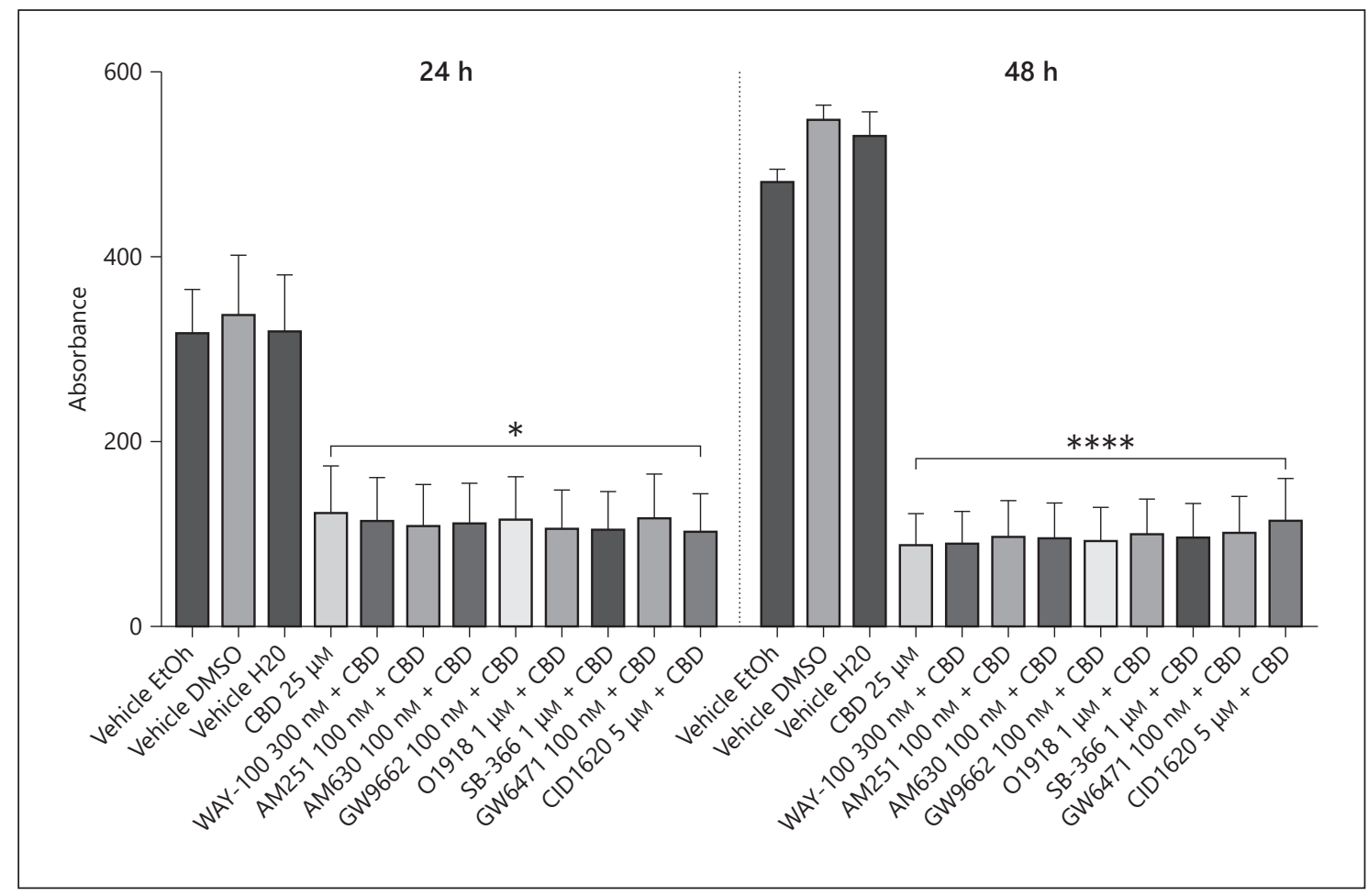

Fig. 2. Effects of CBD (THC Pharm) $25 \mu \mathrm{M}$ on proliferating SKOV-3 cells after 24 or $48 \mathrm{~h}$ in the presence of WAY100635, AM251, AM630, GW9662, O1918, SB366791 GW6471, or CID16020046 ( $n=7$ from 2 separate experiments). Data are displayed as mean \pm SEM absorbance, and CBD + antagonist data were compared against CBD $25 \mu \mathrm{M}$ alone using a one-way ANOVA. ${ }^{*} p \leq 0.05 ;{ }^{* * * *} p \leq 0.0001 . \mathrm{CBD}$, cannabidiol.

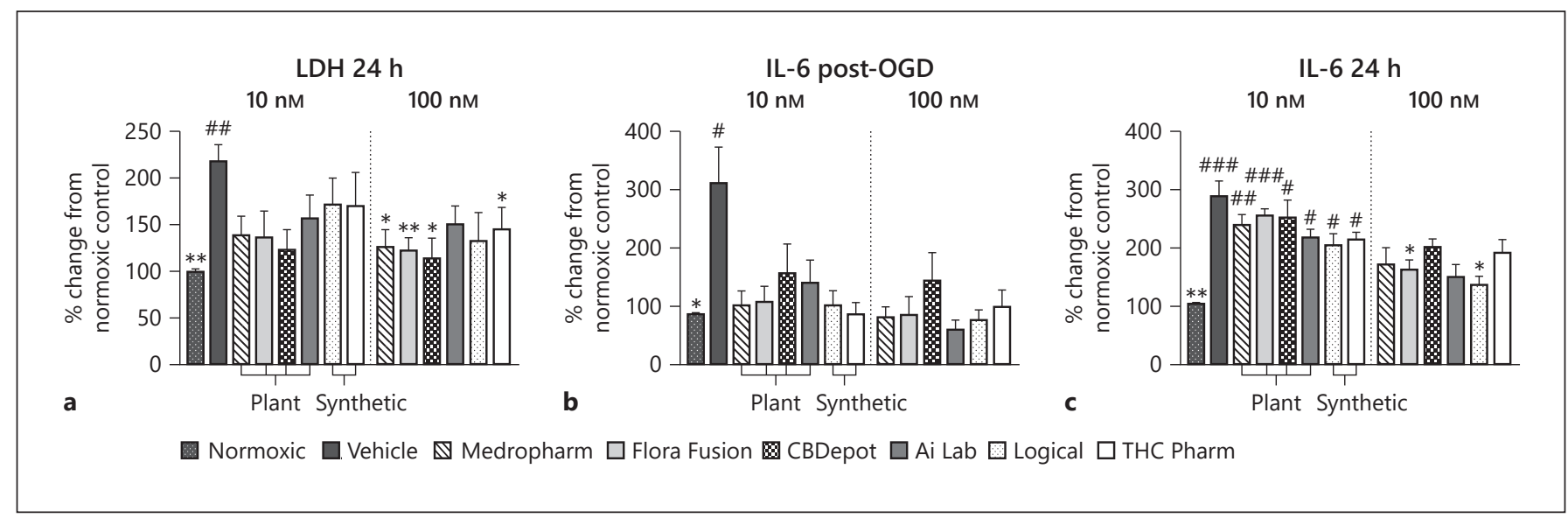

Fig. 3. Effects of CBD in pericytes (p4-6) exposed to 4-h OGD. a Effect of CBD (10 or $100 \mathrm{nM}$ ) on LDH concentrations $24 \mathrm{~h}$ following initiation of OGD ( $n=6$, from 2 separate experiments). Effect of various CBD samples (10 or $100 \mathrm{nM}$ ) on media IL- 6 concentrations immediately after OGD (b), or $24 \mathrm{~h}$ following the initiation of OGD (c) ( $n=6$, from 2 separate experiments). Data are displayed as mean \pm SEM \% change from normoxic control and were compared for statistical significance against the normoxic control (\#) or OGD vehicle $(*)$ using a one-way ANOVA. ${ }^{*} p \leq 0.05$, ${ }^{* *} p \leq 0.01$, and ${ }^{* * *} p \leq 0.001$. CBD, cannabidiol; OGD, oxygen glucose deprivation. 


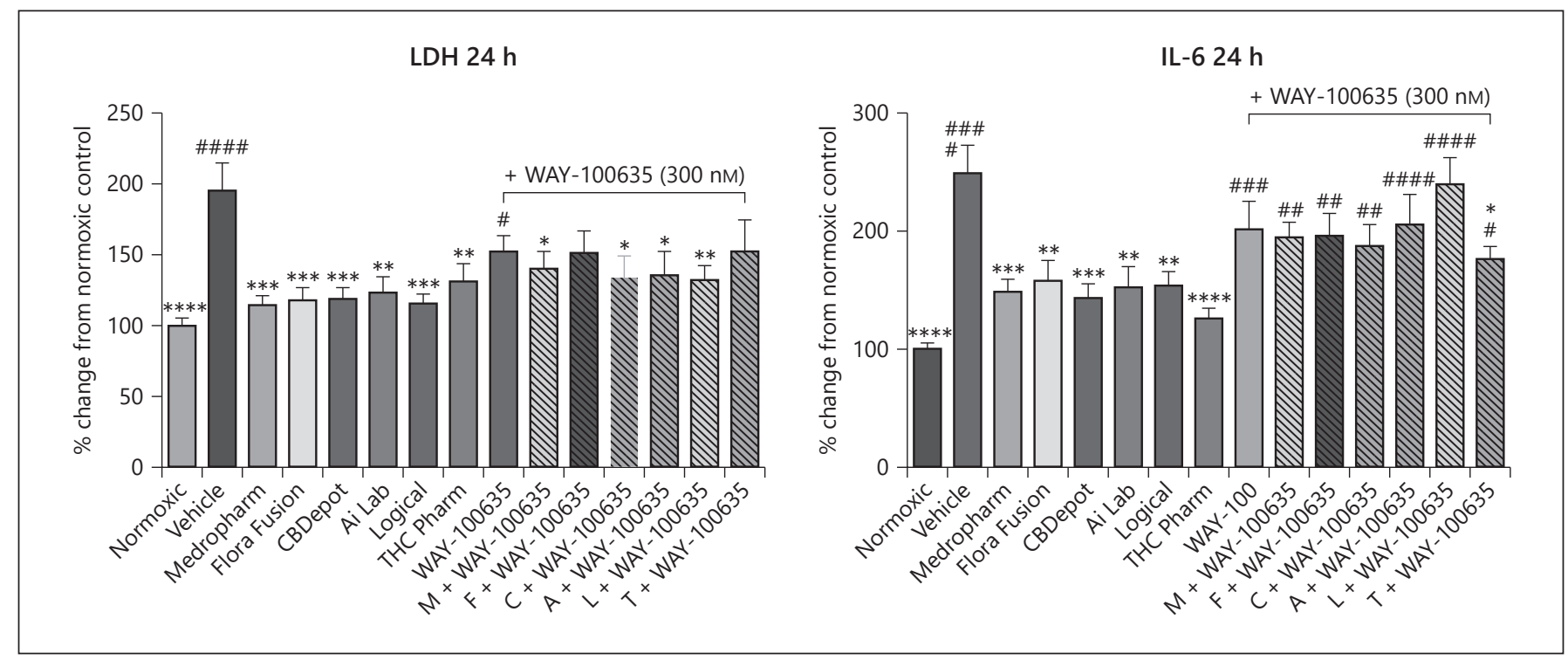

Fig. 4. Effects of natural and synthetic CBD (100 nM) on LDH and IL-6 concentrations in pericytes exposed to 4-h OGD in the presence or absence of the 5HT-1A antagonist WAY-100635 (300 nM). a IL- 6 concentrations 24 h following the initiation of OGD $(n=$ 11-13, from 3 separate experiments). b LDH concentrations in media from $24 \mathrm{~h}$ following initiation of OGD $(n=12-13$, from 3 separate experiments). Data are expressed as mean \pm SEM \% change from normoxic and were compared to normoxic control (\#) or OGD vehicle $\left(^{*}\right)$ using a one-way ANOVA with a multiple comparison. ${ }^{*} p \leq 0.05,{ }^{* *} p \leq 0.01,{ }^{* * *} p \leq 0.001$, and ${ }^{* * * *} p \leq 0.0001$. M, Medropharm; F, Flora Fusion; C, CBDepot; A, Ai Lab; L, Logical; T, THC Pharm; CBD, cannabidiol; OGD, oxygen glucose deprivation. also not significantly raised relative to the normoxic control in the presence of CBD (100 nM). No statistical differences were present between CBD samples at their equivalent concentrations and time points.

$\mathrm{LDH}$ activity was also not significantly raised above normoxic control in the presence of CBD (24 h; Fig. 3c). At $100 \mathrm{nM}$, Medropharm $(-91 \% \pm 18, p<0.05)$, Flora Fusion $(-96 \% \pm 9, p<0.01)$, CBDepot $(-104 \% \pm 19, p<$ $0.05)$, and THC Pharm $(-73 \% \pm 18, p<0.05)$ also significantly reduced LDH activity relative to the OGD vehicle.

CBD $100 \mathrm{nM}$ was coapplied with the $5 \mathrm{HT}_{1 \mathrm{~A}}$ antagonist WAY-100635 [33]. As before, IL-6 secretion was blunted by CBD (Fig. 4a, 50-63\% relative to vehicle). However, in the presence of WAY-100635, this effect was reduced such that an increase in IL-6 secretion was observed (Fig. 4a). There was no obvious effect of WAY-100635 on LDH activity (Fig. 4b).

\section{CBD Effects on Caco-2 Barrier Integrity following Inflammation}

Following an inflammatory protocol, barrier resistance was reduced by $\sim 30 \%$, indicating increased permeability [36]. When analyzed as the total effect over time (AUC) $\left(\% \cdot \mathrm{min}^{-1}\right)$, all CBD samples increased TEER fol- lowing inflammation relative to their vehicle control (Fig. 5b-f; Table 1 for pooled data). No statistical differences were present between CBD samples in their ability to restore intestinal permeability. As before (Fig. 5), when $\mathrm{CBD}$ was administered alone, it successfully increased the recovery of TEER values following a 24-h inflammatory protocol. However, when CBD was coadministered with AM251 (1 $\mu \mathrm{M})$, this effect was inhibited (Fig. 6).

\section{Discussion}

The aim of this study was to investigate whether CBD derived from different sources (natural or synthetic) would behave similarly in 3 human cell models of disease. Across all experiments, all CBD samples at their respective concentrations and time points produced very similar effects. Whilst some minor variability existed in the magnitude of effect and percentage purity of CBD samples, none of these differences manifested in one particular sample of CBD being superior. These data support our hypothesis that there is no pharmacological difference between purified natural and synthetic CBD in vitro. 


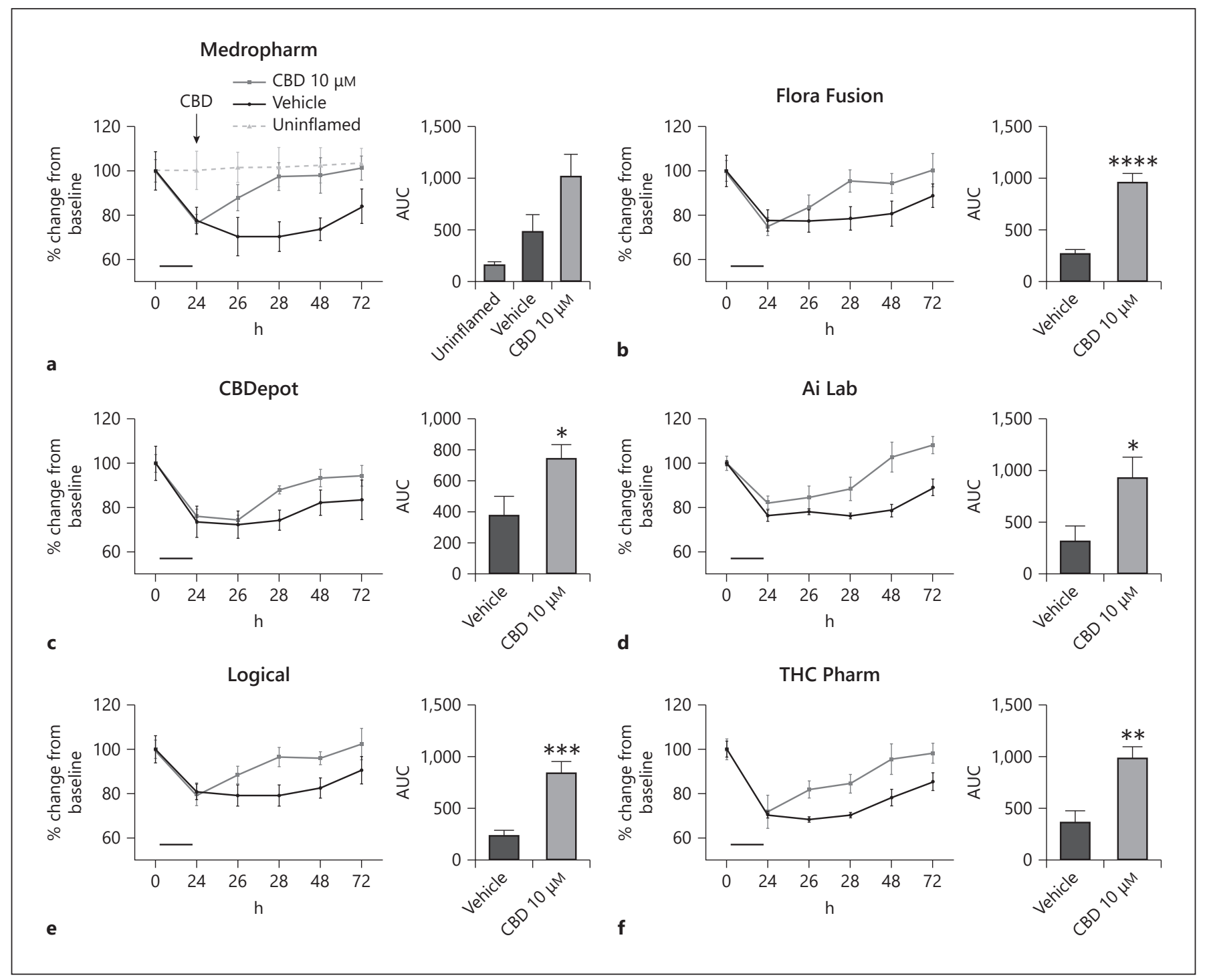

Fig. 5. Effects of natural (a-d) and synthetic $\mathrm{CBD}(\mathbf{e}, \mathbf{f})$ on intestinal permeability following a 24 -h inflammatory protocol (IFN $\gamma$ and TNFa, shown as the solid bar) in differentiated Caco-2 cells. Data are displayed as mean \pm SEM \% change from baseline inserts ( $n=5-6$, from 3 separate experiments). Time course data are dis-

CBD has antiproliferative $[37,38]$ and apoptotic features [39] in multiple cancer cell lines. We explored the effects of CBD in the ovarian cancer cell line SKOV-3 and whether the CBD origin influenced results. Ten micromolar CBD reduced cell viability in proliferating cells, and $50 \mu \mathrm{M} \mathrm{CBD}$ was cytotoxic in proliferating and confluent cells. The effects of CBD were greater at $48 \mathrm{~h}$. None of the purified CBD samples differed in their efficacy across concentrations or time points, suggesting regardless of how $\mathrm{CBD}$ is produced, its antiproliferative/cytotoxic ef- played as AUC and analyzed using a one-way ANOVA. CBD samples were compared to vehicle or each other using a multiple comparison ${ }^{*} p \leq 0.05,{ }^{* *} p \leq 0.01$, and ${ }^{* * *} p \leq 0.001$. AUC, area under the curve; $\mathrm{CBD}$, cannabidiol.

fects remain the same, at least in the SKOV-3 cell line. Since the effects of CBD were not attenuated by the range of antagonists used and the high concentration of CBD required, it is likely this is a nonreceptor-mediated mechanism, for example, on mitochondria membrane potential and/or ROS generation [40], which should be investigated in future experiments.

CBD possesses several properties that could be useful in treating ischemia and reperfusion injuries including modulation of intracellular calcium [41], attenuating 


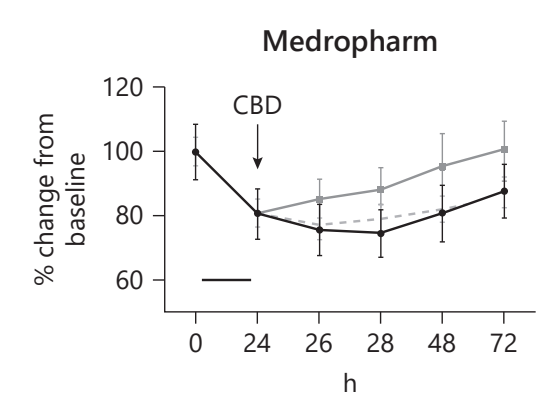

a

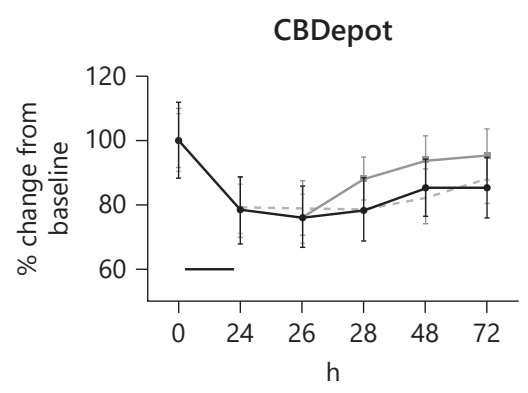

c

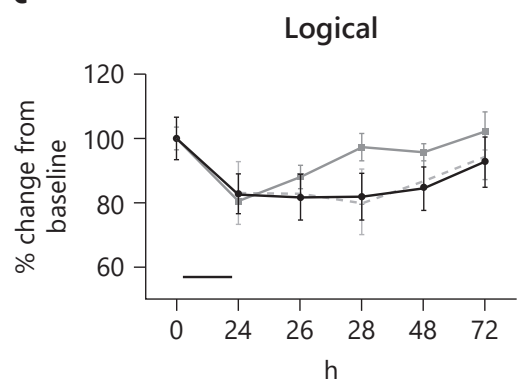

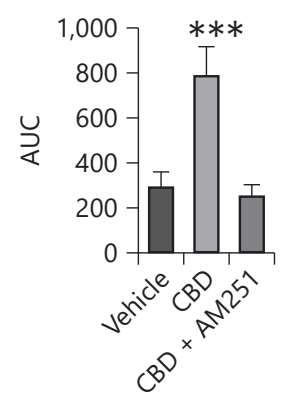
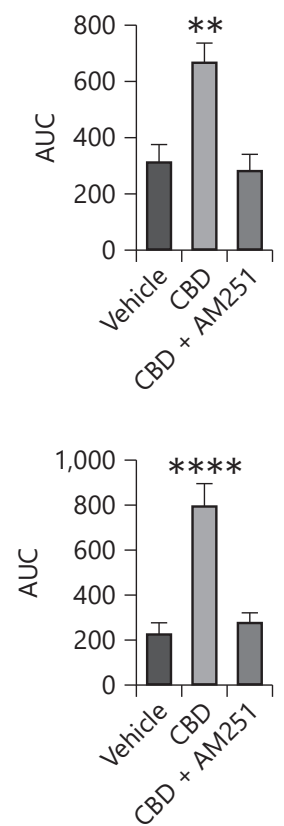

Flora Fusion

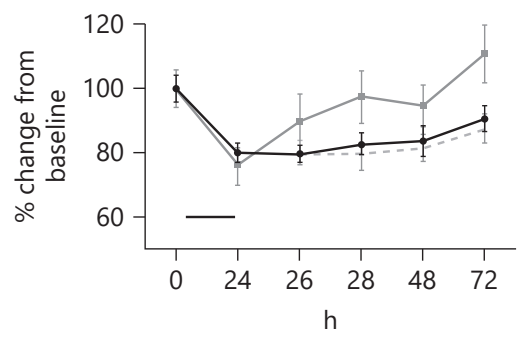

b

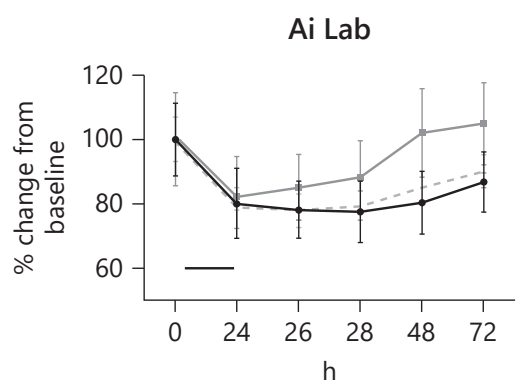

d

THC Pharm

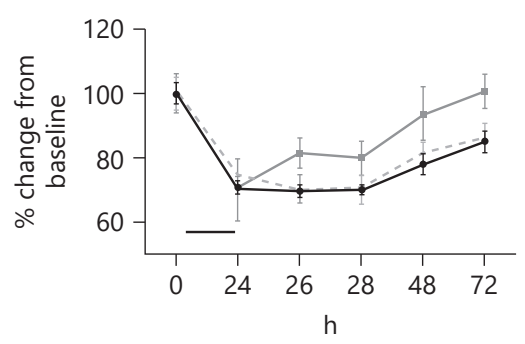

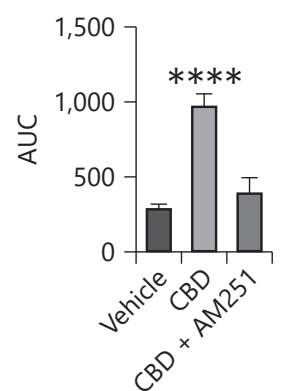
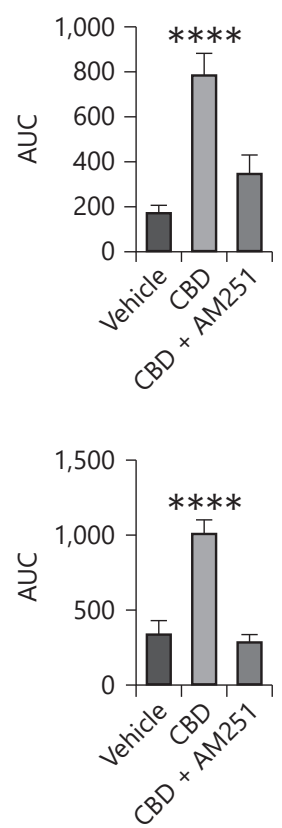

e

$\mathbf{f}$

Time course data are displayed as AUC and analyzed using a oneway ANOVA. CBD samples were compared to vehicle or each other using a multiple comparison ${ }^{* *} p \leq 0.01,{ }^{* * *} p \leq 0.001$, and ${ }^{* * * *} p \leq 0.0001$. AUC, area under the curve; CBD, cannabidiol.

activate $5 \mathrm{HT}_{1 \mathrm{~A}}$, which is the molecular target underpinning many therapeutic effects including stroke, anxiety, and pain. As far as we are aware, this is the first study showing the protective properties of CBD in pericytes exposed to OGD/R injury.

As we have previously shown [36], Caco-2 barrier integrity could be restored by administering CBD to the apical compartment of transwell inserts following a 24-h inflammatory protocol. Further work by our laboratory demonstrated these effects of CBD extend to human co- 
lon explants exposed to inflammation [45]. In both studies, the ability of CBD to restore barrier integrity was inhibited by $\mathrm{CB}_{1}$ antagonism $[36,45]$. Most recently, we showed that CBD $(600 \mathrm{mg})$ was protective against aspirin-induced permeability increases in healthy volunteers in vivo [45]. In this present set of experiments, all CBD samples restored barrier integrity of differentiated Caco2 cells following inflammation to a similar extent, and the effects on barrier integrity were reduced by antagonism of the $\mathrm{CB}_{1}$ receptor for all $\mathrm{CBD}$ samples. These results suggest natural and synthetic $\mathrm{CBDs}$ have similar pharmacological effects in a cellular model of acutely inflamed small intestine.

One caveat to consider is that natural CBD can contain other phytocannabinoids, albeit in small amounts (see Table 1). However, the concentration of these compounds would be moderate with high concentrations of CBD. For example, the most abundant contaminant was CBDV $(0.6 \%)$. At $50 \mu \mathrm{M}$, the corresponding concentration of CBDV would be $\sim 300 \mathrm{nM}$. The CBDV pharmacology is not well characterized, but binding assays demonstrated a $K_{\mathrm{i}}$ of $14.7 \mu \mathrm{M}$ at $\mathrm{CB}_{1}$ and $570 \mathrm{nM}$ at $\mathrm{CB}_{2}$ [46]. We believe it is unlikely that the small concentrations of CBDV, or indeed other Cannabis constituents, influenced our results at a pharmacodynamic level. Whether there are pharmacokinetic interactions between these compounds in vivo remains to be tested.

This study demonstrates for the first time that the anticancer, neuroprotective, and intestinal barrier protective properties of purified CBD are similar regardless of the source from which CBD is derived. From a pharmacological perspective, where a molecular target is implicated (i.e., $5 \mathrm{HT}_{1 \mathrm{~A}}$ in stroke and $\mathrm{CB}_{1}$ in gut permeability), the effects of CBD were similar. This suggests that any beneficial effects that could be achieved in a clinical set- ting for purified CBD are likely to be similar at a pharmacodynamic level. Based on this analysis, the economic, environmental impact, purity, reliability, and consistency of CBD, either natural or synthetic, as well as the ultimate pharmaceutical preparation, should all be considered above the starting origin of the CBD in the development of new CBD medicines.

\section{Statement of Ethics}

The cell line used in this study was purchased from the European Collection of Authenticated Cell Cultures (SKOV-3 and Caco- 2 cells) and ScienceCell, USA (Human brain vascular pericytes). Ethical approval for the use of these cells is not required in accordance with local/national guidelines.

\section{Conflict of Interest Statement}

S.E.O'S. is an independent consultant and paid scientific advisor to Artelo Biosciences.

\section{Funding Sources}

R.M.'s PhD was funded by Artelo Biosciences, and S.E.O'S. is a paid scientific advisor to Artelo Biosciences.

\section{Author Contributions}

R.F.M. and D.J.W. performed the analysis and interpretation described in the manuscript. T.J.E. and S.E.O'S. made substantial contribution to the conception and design of the work. All authors were involved in drafting and reviewing the work and approved the final manuscript.

\section{References}

1 Blessing EM, Steenkamp MM, Manzanares J, Marmar CR. Cannabidiol as a potential treatment for anxiety disorders. Neurotherapeutics. 2015 Oct 4;12(4):825-36.

2 Cherniakov I, Izgelov D, Domb AJ, Hoffman A. The effect of pro nanolipospheres (PNL) formulation containing natural absorption enhancers on the oral bioavailability of delta9-tetrahydrocannabinol (THC) and cannabidiol (CBD) in a rat model. Eur J Pharm Sci. 2017 Nov 15;109:21-30.

3 Devinsky O, Cilio MR, Cross H, FernandezRuiz J, French J, Hill C, et al. Cannabidiol: pharmacology and potential therapeutic role in epilepsy and other neuropsychiatric disorders. Epilepsia. 2014 Jun;55(6):791-802.
4 Dumitru CA, Sandalcioglu IE, Karsak M. Cannabinoids in glioblastoma therapy: new applications for old drugs. Front Mol Neurosci. 2018;11:159.

5 Gofshteyn JS, Wilfong A, Devinsky O, Bluvstein J, Charuta J, Ciliberto MA, et al. Cannabidiol as a potential treatment for febrile infection-related epilepsy syndrome (FIRES) in the acute and chronic phases. J Child Neurol. 2017;32(1):35-40.

6 Hayakawa K, Mishima K, Fujiwara M. Therapeutic potential of non-psychotropic cannabidiol in ischemic stroke. Pharmaceuticals. 2010;3(7):2197-212.

7 Śledziński P, Zeyland J, Słomski R, Nowak A. The current state and future perspectives of cannabinoids in cancer biology. Cancer Med. 2018 Mar;7(3):5859-75.

8 Syed YY, McKeage K, Scott LJ. Delta-9-tetrahydrocannabinol/cannabidiol (sativex ${ }^{\oplus}$ ): a review of its use in patients with moderate to severe spasticity due to multiple sclerosis. Drugs. 2014 Apr 27;74(5):563-78.

9 Freeman TP, Hindocha C, Green SF, Bloomfield MAP. Medicinal use of cannabis based products and cannabinoids. BMJ. 2019 Apr 4; 365:11141.

10 Ibeas Bih C, Chen T, Nunn AV, Bazelot M, Dallas M, Whalley BJ. Molecular targets of cannabidiol in neurological disorders. Neurotherapeutics. 2015 Oct 12;12(4):699_ 730. 
11 Elsaid S, Le Foll B. The complexity of pharmacology of cannabidiol (CBD) and its implications in the treatment of brain disorders. Neuropsychopharmacology. 2020;45:229-30.

12 de Mello Schier AR, de Oliveira Ribeiro NP, Coutinho DS, Machado S, Arias-Carrión O, Crippa JA, et al. Antidepressant-like and anxiolytic-like effects of cannabidiol: a chemical compound of cannabis sativa. CNS Neurol Disord Drug Targets. 2014;13(6):953-60.

13 Hayakawa K, Mishima K, Nozako M, Hazekawa M, Irie K, Fujioka M, et al. Delayed treatment with cannabidiol has a cerebroprotective action via a cannabinoid receptor-independent myeloperoxidase-inhibiting mechanism. J Neurochem. 2007 Sep 1;102(5): 1488-96.

14 Rock EM, Bolognini D, Limebeer CL, Cascio MG, Anavi-Goffer S, Fletcher PJ, et al. Cannabidiol, a non-psychotropic component of cannabis, attenuates vomiting and nausealike behaviour via indirect agonism of 5-HT(1A) somatodendritic autoreceptors in the dorsal raphe nucleus. Br J Pharmacol. 2012 Apr;165(8):2620-34.

15 Costa B, Giagnoni G, Franke C, Trovato AE, Colleoni M. Vanilloid TRPV1 receptor mediates the antihyperalgesic effect of the nonpsychoactive cannabinoid, cannabidiol, in a rat model of acute inflammation. Br J Pharmacol. 2004 Sep;143(2):247-50.

16 Ramer R, Heinemann K, Merkord J, Rohde H, Salamon A, Linnebacher M, et al. COX-2 and PPAR- $\gamma$ confer cannabidiol-induced apoptosis of human lung cancer cells. Mol Cancer Ther. 2013 Jan 1;12(1):69-82.

17 Alhamoruni A, Wright KL, Larvin M, O'Sullivan SE. Cannabinoids mediate opposing effects on inflammation-induced intestinal permeability. Br J Pharmacol. 2012 Apr 1; 165(8):2598-610.

18 Couch DG, Cook H, Ortori C, Barrett D, Lund JN, O'Sullivan SE. Palmitoylethanolamide and cannabidiol prevent inflammation-induced hyperpermeability of the human gut in vitro and in vivo-a randomized, placebo-controlled, double-blind controlled trial. Inflamm Bowel Dis. 2019 May 4;25(6): 1006-18.

19 Jung B, Lee JK, Kim J, Kang EK, Han SY, Lee $\mathrm{H}$, et al. Synthetic strategies for (-)-cannabidiol and its structural analogs. Chem Asian J. 2019 Nov 4;14(21):3749-62.

20 Gallily R, Yekhtin Z, Hanuš LO. Overcoming the bell-shaped dose-response of cannabidiol by using cannabis extract enriched in cannabidiol. Pharm Pharmacol. 2015;6(2):75-85.

21 Pagano E, Capasso R, Piscitelli F, Romano B, Parisi OA, Finizio $S$, et al. An orally active cannabis extract with high content in cannabidiol attenuates chemically-induced intestinal inflammation and hypermotility in the mouse. Front Pharmacol. 2016;7:341.
22 Scott KA, Dalgleish AG, Liu WM. The combination of cannabidiol and $\Delta 9$ tetrahydrocannabinol enhances the anticancer effects of radiation in an orthotopic murine glioma model. Mol Cancer Ther. 2014 Dec 1;13(12):2955-67.

23 Ligresti A, Moriello AS, Starowicz K, Matias I, Pisanti S, De Petrocellis L, et al. Antitumor activity of plant cannabinoids with emphasis on the effect of cannabidiol on human breast carcinoma. J Pharmacol Exp Ther. 2006; 318(3):1375-87.

24 Raup-Konsavage WM, Carkaci-Salli N, Greenland K, Gearhart R, Vrana KE. Cannabidiol (CBD) oil does not display an entourage effect in reducing cancer cell viability in vitro. Med Cannabis Cannabinoids. 2020; 3(2):95-102.

25 von Wrede R, Moskau-Hartmann S, Amarell N, Surges R, Erich Elger C, Helmstaedter C. Plant derived versus synthetic cannabidiol: wishes and commitment of epilepsy patients. Seizure. 2020 Aug 1;80:92-5.

26 Montoya Z, Conroy M, Vanden Heuvel BD, Pauli CS, Park SH. Cannabis contaminants limit pharmacological use of cannabidiol. Front Pharmacol. 2020;11:571832.

27 Klotz KA, Grob D, Hirsch M, Metternich B, Schulze-Bonhage A, Jacobs J. Efficacy and tolerance of synthetic cannabidiol for treatment of drug resistant epilepsy. Front Neurol. 2019; 10:1313.

28 Wheless JW, Dlugos D, Miller I, Oh DA, Parikh N, Phillips S, et al. Pharmacokinetics and tolerability of multiple doses of pharmaceutical-grade synthetic cannabidiol in pediatric patients with treatment-resistant epilepsy. CNS Drugs. 2019 Jun 5;33(6):593-604.

29 Devinsky O, Patel AD, Cross JH, Villanueva V, Wirrell EC, Privitera M, et al. Effect of cannabidiol on drop seizures in the lennoxgastaut syndrome. N Engl J Med. 2018 May 17;378(20):1888-97.

30 Thiele EA, Marsh ED, French JA, Mazurkiewicz-Beldzinska M, Benbadis SR, Joshi C, et al. Cannabidiol in patients with seizures associated with lennox-gastaut syndrome (GWPCARE4): a randomised, double-blind, placebo-controlled phase 3 trial. Lancet. 2018 Mar 17;391(10125):1085-96.

31 Devinsky O, Patel AD, Thiele EA, Wong MH Appleton R, Harden CL, et al. Randomized, dose-ranging safety trial of cannabidiol in dravet syndrome. Neurology. 2018 Apr 3; 90(14):e1204-11.

32 Szaflarski JP, Bebin EM, Comi AM, Patel AD, Joshi C, Checketts D, et al. Long-term safety and treatment effects of cannabidiol in children and adults with treatment-resistant epilepsies: expanded access program results. Epilepsia. 2018;59(8):1540-8.

33 Hind WH, England TJ, O'Sullivan SE. Cannabidiol protects an in vitro model of the blood-brain barrier from oxygen-glucose deprivation via PPAR $\gamma$ and 5-HT1A receptors. Br J Pharmacol. 2016 Mar;173(5):81525.
34 Stone NL, England TJ, O’Sullivan SE. A novel transwell blood brain barrier model using primary human cells. Front Cell Neurosci. 2019 Jun 6;13:230

35 Wells CL, van de Westerlo EM, Jechorek RP, Haines HM, Erlandsen SL. Cytochalasin-induced actin disruption of polarized enterocytes can augment internalization of bacteria. Infect Immun. 1998 Jun;66(6):2410-9.

36 Alhamoruni A, Wright KL, Larvin M, O'Sullivan SE. Cannabinoids mediate opposing effects on inflammation-induced intestinal permeability. Br J Pharmacol. 2012 Apr 1; 165(8):2598-610

37 De Petrocellis L, Ligresti A, Schiano Moriello A, Iappelli M, Verde R, Stott CG, et al. NonTHC cannabinoids inhibit prostate carcinoma growth in vitro and in vivo: pro-apoptotic effects and underlying mechanisms. $\mathrm{Br} \mathrm{J}$ Pharmacol. 2013;168(1):79-102.

38 Ligresti A, Moriello AS, Starowicz K, Matias I, Pisanti S, De Petrocellis L, et al. Antitumor activity of plant cannabinoids with emphasis on the effect of cannabidiol on human breast carcinoma. J Pharmacol Exp Ther. 2006; 318(3):1375-87.

39 Ramer R, Heinemann K, Merkord J, Rohde H, Salamon A, Linnebacher M, et al. COX-2 and PPAR- $\gamma$ confer cannabidiol-induced apoptosis of human lung cancer cells. Mol Cancer Ther. 2013 Jan 1;12(1):69-82.

40 Massi P, Solinas M, Cinquina V, Parolaro D. Cannabidiol as potential anticancer drug. $\mathrm{Br} J$ Clin Pharmacol. 2013 Feb;75(2):303-12.

41 Ryan D, Drysdale AJ, Lafourcade C, Pertwee RG, Platt B. Cannabidiol targets mitochondria to regulate intracellular Ca2+ levels. J Neurosci. 2009 Feb 18;29(7):2053-63.

42 Sun S, Hu F, Wu J, Zhang S. Cannabidiol attenuates OGD/R-induced damage by enhancing mitochondrial bioenergetics and modulating glucose metabolism via pentosephosphate pathway in hippocampal neurons. Redox Biol. 2017;11:577-85.

43 Hampson AJ, Grimaldi M, Axelrod J, Wink D. Cannabidiol and (-)delta9-tetrahydrocannabinol are neuroprotective antioxidants. Proc Natl Acad Sci U S A. 1998 Jul 7;95(14): 8268-73.

44 Mishima K, Hayakawa K, Abe K, Ikeda T, Egashira N, Iwasaki K, et al. Cannabidiol prevents cerebral infarction via a serotonergic 5-hydroxytryptamine1A receptor-dependent mechanism. Stroke. 2005;36(5):1077-82.

45 Couch DG, Cook H, Ortori C, Barrett D, Lund JN, O'Sullivan SE. Palmitoylethanolamide and cannabidiol prevent inflammation-induced hyperpermeability of the human gut in vitro and in vivo-a randomized, placebo-controlled, double-blind controlled trial. Inflamm Bowel Dis. 2019 May 4;25(6):1006-18.

46 Rosenthaler S, Pöhn B, Kolmanz C, Huu CN, Krewenka C, Huber A, et al. Differences in receptor binding affinity of several phytocannabinoids do not explain their effects on neural cell cultures. Neurotoxicol Teratol. 2014 Nov 1;46:49-56. 